\title{
BMJ Open Intersectional stigma and its impact on HIV prevention and care among MSM and WSW in sub-Saharan African countries: a protocol for a scoping review
}

\author{
Gamji M'Rabiu Abubakari (10 , ${ }^{1}$ Debbie Dada, ${ }^{2,3}$ Jemal Nur, ${ }^{3}$ DeAnne Turner, ${ }^{4}$ \\ Amma Otchere, ${ }^{2}$ Leonne Tanis, ${ }^{2}$ Zhao Ni, ${ }^{5}$ Ibrahim Wunpini Mashoud, ${ }^{6}$ \\ Kate Nyhan, ${ }^{7}$ Laura Nyblade, ${ }^{8}$ LaRon E Nelson ${ }^{2,3}$
}

To cite: Abubakari GM'R, Dada D, Nur J, et al. Intersectional stigma and its impact on HIV prevention and care among MSM and WSW in sub-Saharan African countries: a protocol for a scoping review. BMJ Open 2021;11:e047280. doi:10.1136/ bmjopen-2020-047280

- Prepublication history and additional supplemental material for this paper are available online. To view these files, please visit the journal online. (http://dx.doi.org/10.1136/ bmjopen-2020-047280).

Received 25 November 2020 Accepted 25 May 2021

D) Check for updates

(c) Author(s) (or their employer(s)) 2021. Re-use permitted under CC BY-NC. No commercial re-use. See rights and permissions. Published by BMJ.

For numbered affiliations see end of article.

\section{Correspondence to} Dr Gamji M'Rabiu Abubakari; mohammed-rabiu.abubakari@ yale.edu

\section{ABSTRACT}

Introduction Research has established that various forms of stigma (HIV stigma, gender non-conforming stigma and same-gender sex stigma) exist across Sub-Saharan Africa and have consequences for the utilisation of HIV prevention and care services. Stigmas are typically investigated in HIV literature individually or through investigating individual populations and the various stigmas they may face. The concept of intersectionality highlights the interconnected nature of social categorisations and their ability to create interdependent systems of discrimination based on gender, race, sexuality and so on. Drawing from perspectives on intersectionality, intersectional stigma denotes the convergence of multiple marginalised identities within an individual or a group, the experiences of stigma associated with these identities as well as the synergistic impact of these experiences on health and well-being. With respect to HIV, public health scholars can examine the impacts of intersectional stigmas on HIV prevention and care utilisation.

Methods and analysis Reviewers will search systematically through MEDLINE, Global Health, Embase, Scopus, Web of Science Core Collection and Africa Index Medicus and citations for quantitative studies, qualitative studies and grey literature that include data on stigma and HIV among men who have sex with men and women who have sex with women in Sub-Saharan Africa. Eligible studies will include primary or secondary data on stigma related to HIV risk factors experienced by this population. Studies will be written in French or English and be published between January 1991 and November 2020. All screening and data extraction will be performed in duplicate, and if discrepancies arise, they will be settled by GM'RA, LEN, DD or AO. Findings from this study will be reported according to the Preferred Reporting Items for Systematic reviews and Meta-Analyses extension for Scoping Reviews.

Ethics and dissemination Ethics approval is not required as there will be no human participants and no protected data will be used in this study. We will disseminate findings through peer-reviewed manuscripts, conferences and webinars.

\section{Strengths and limitations of this study}

- This scoping review is reinforced by the comprehensive search strategy designed by an experienced public health librarian, peer-reviewed by an independent medical librarian and supplemented with citation chaining.

- The novel approach to this research topic is a strength as it will enable us to highlight the current status of intersectional stigma as a key determinant of access to HIV prevention and care among key populations.

- A limitation of this review is the focus on SubSaharan Africa, as it restricts generalisability to the experiences of men who have sex with men and women who have sex with women globally.

\section{INTRODUCTION}

HIV remains one of the leading causes of death worldwide, with the majority of global cases concentrated in sub-Saharan Africa. ${ }^{12}$ Though sub-Saharan Africa comprises $12 \%$ of the world population, this region accounted for $75 \%$ of HIV-related deaths and $65 \%$ of new HIV infections globally in 2017. ${ }^{2}$ Rates of HIV infections are particularly high in Eastern and Southern Africa, with nine countries in this region accounting for nearly $50 \%$ of the global total of people living with HIV. ${ }^{3}$ In any global context, men who have sex with men (MSM) stand at a heightened risk for HIV infection. ${ }^{45}$ Within sub-Saharan Africa, HIV prevalence among MSM is three times as high as HIV prevalence in the general male population. ${ }^{5}$ Also, despite the disproportionate burden of HIV among women in sub-Saharan Africa, limited research examines the prevalence of HIV among women who have sex with women (WSW) in this region. ${ }^{36}$ There 
exists a long-standing assumption that WSW do not face a high risk of HIV infection, yet some existing research has drawn contrary conclusions, supporting the need for investigating the risks of HIV infection and barriers to HIV prevention and care services among WSW in sub-Saharan Africa ${ }^{6-8}$ While MSM and WSW populations constitute a small percentage of the overall population, they have an extremely high risk for HIV transmission with estimated prevalence in Sub-Saharan countries ranging from $12 \%$ to as high as $30 \% .^{9}$ MSM and WSW face several barriers to HIV prevention and treatment, from community-wide issues (eg, inadequate financial resources, lack of convenient access to healthcare clinics) to specific barriers that arise as a result of their HIV status and/or from their identity as MSM or WSW (eg, stigma). ${ }^{5} 710$

Stigma emanates from a construct of psychological, social and societal factors that engender and enforce inequalities between groups of people. ${ }^{11}$ The socialecological model emphasises the multilayered manner in which people conceive and enact stigma as well as the important role power structures play in maintaining it. ${ }^{12}$ Stigmas are commonly associated with certain sociodemographic characteristics, behaviours, experiences and health conditions. Stigma can be manifested and experienced on the individual, interpersonal, institutional and societal level. Drivers (such as stereotypes and lack of awareness) cause stigma on an individual level, while facilitators (such as laws, culture and gender norms) cause it at a societal level. ${ }^{13}$

Intersectional stigma occurs when an individual or group experience(s) multiple stigmas that are not only overlapping but also coconstitutive. It denotes the synergistic effect produced by systems of oppression at the intersection of these stigmatised identities, behaviours and/or conditions on well-being and health. ${ }^{14-16}$ HIV scholars predominantly examine stigma and marginalised identities in a piecemeal way by mainly focusing on single factors such as gender, sexual orientation, profession, race, socioeconomic status and so on. ${ }^{17-19}$ MSM in Sub-Saharan Africa have been reported to experience intersectional stigma with regards to same-sex attraction, gender-non-conforming behaviour, actual or perceived HIV statusand so on. ${ }^{20}$ At healthcare facilities, MSM experience treatment refusal, verbal abuse and avoidance behaviours (like double gloving) and on the social level, they experience physical and verbal harassment, social isolation and discrimination. ${ }^{21-24}$ For the sake of preserving interpersonal relationships, many MSM avoid HIV-related care out of fear of others suspecting that they have sex with men and this anticipated stigma is further compounded by a fear of discriminatory treatment at healthcare facilities. ${ }^{25}{ }^{26}$ These are just some of the reasons for which fear of stigmatisation and discrimination are key determinants of voluntary HIV testing, care seeking and linkage to care among MSM. ${ }^{27}{ }^{28}$ By viewing stigma through an intersectional lens, researchers are able to gain a more comprehensive view of the impact of HIV/AIDS on lived experience, to identify synergies between risk factors that may allow for development of more impactful and efficient solutions and to develop multifaceted solutions.

Different forms of stigma, while often analysed individually, do not exist singularly. While MSM and WSW experience intersectional forms of stigma, the ways in which intersectional stigma affects health outcomes for these groups remain poorly understood. ${ }^{14}$ In Sub-Saharan Africa, specifically, there are a limited number of studies on intersectional stigma and its implications for HIVrelated health outcomes. The primary objective of this study is to assess the state of science with regards to HIVrelated stigma research on MSM and WSW populations in Sub-Saharan Africa. It aims to investigate the common presentations of stigma in HIV research and how often stigma is discussed and conceptualised in research as an intersectional experience. The secondary objective of this study is to evaluate how the experience of intersectional stigma is shown to affect HIV prevention and care for MSM and WSW in literature. Overall, this study intends to provide an overview of intersectional experiences of stigma among MSM and WSW and its effects on HIVrelated health outcomes.

\section{METHODS AND ANALYSIS}

To develop the protocol for this scoping review, we used the guideline for scoping reviews; the Preferred Reporting Items for Systematic Reviews and Meta-Analyses extension for Scoping Reviews (PRISMA-ScR). ${ }^{29}$ As explained by Tricco $e t a l,{ }^{29}$ before a scoping review, the research team needs to develop a protocol to explain the intent and processes of the scoping review. ${ }^{29}$ Such a protocol, when reviewed and published, will ensure transparency of the process and avoidance of duplication of efforts.

\section{Ethics and dissemination}

There will be no human participants and no data collection in this study. As such, ethics approval is not required. ${ }^{30}$ The findings from this study will be disseminated through conferences, webinars, peer-reviewed manuscripts and could inform the policies and practices of government health agencies and healthcare facilities.

\section{Patient and public involvement}

We will not engage MSM and WSW when conducting this scoping review. We, however, anticipate to disseminate findings to the public, with special attention to audiences involved in HIV research and practice in order to inform decision-making in healthcare practice and policy. ${ }^{31}$

\section{Criteria for study inclusion}

For an article to qualify for inclusion in this scoping review, it must discuss at least one or more types of stigma related to HIV risk factors, prevention, intervention or care. The study must also present primary or secondary data involving MSM or WSW and be conducted in one or more sub-Saharan African countries. The United 
Nations defines sub-Saharan African countries to include all countries south of the Sahara, namely, Angola, Benin, Botswana, Burkina Faso, Burundi, Cameroon, Central African Republic, Chad, Congo, Cote d'Ivoire, Eritrea, Ethiopia, Gabon, Gambia, Ghana, Guinea, Guinea-Bissau, Kenya, Lesotho, Liberia, Madagascar, Malawi, Mali, Mauritania, Mauritius, Mozambique, Namibia, Niger, Nigeria, Rwanda, Senegal, Sierra Leone, Somalia, South Africa, United Republic of Tanzania, Togo, Uganda, Zaire, Zambia and Zimbabwe. ${ }^{32}$ To ensure an extensive inclusion of articles from as many parts of sub-Saharan Africa as possible, we will include studies written in English and French.

As literature that evaluates stigma related to HIV prevention and care among MSM and WSW in an intersectional manner remains limited, in order to meet our primary objective of evaluating how stigma is discussed in HIV research, we will also include studies that examine a single stigma in our analysis. However, these single-stigma studies will not be included in the full data extraction process (only basic information and the type of stigma studied will be extracted) and will instead only be used to assess what proportion of the literature evaluates stigma intersectionally. ${ }^{33}$ Keeping articles that discuss single stigmas will enable the team to quantify and compare studies on intersectional stigma with those on individual stigmas. We will exclude studies published before 1991 as we identified that the first peer-reviewed journal article focused on intersectional HIV stigma was published in this year. We will exclude studies that combine data from sub-Saharan African countries with data from other countries in a way that the team cannot extract the data on sub-Saharan African countries distinctively.

\section{Types of studies}

Both experimental and observational studies will be considered in this review. Studies will not be excluded based on methodological approaches; quantitative, qualitative and mixed methods studies will be considered. Articles synthesising existing literature, such as reviews, will not be included in analysis but will be used for citation chaining.

\section{Search strategy}

\section{Identifying sources}

Author KN, a research librarian for the Yale University School of Public Health, will lead the search for articles; authors GM'RA, LEN and DD will support KN in identifying sources of grey literature from reports of organisations that examine HIV in sub-Saharan African countries.

\section{Electronic database searching}

We will search for relevant documents in key public health sources MEDLINE (Ovid), Global Health (Ovid) and Embase (Ovid) and bibliographic databases that index content across disciplines: Scopus, Web of Science Core Collection (as licensed at Yale). We will also search regional literature databases: Africa-Wide Information and Africa Index Medicus (through Global Index Medicus). See, for example, online supplemental file 1.

\section{Grey literature searching}

To identify other pertinent documents not found in the electronic search of bibliographic databases, we will search for theses, dissertations and conference papers in the databases ProQuest Dissertations and Theses Global, Open Access Theses and Dissertations and Networked Digital Library of Theses and Dissertations. We will also search on organisational websites, such as the United Nations Program on HIV/AIDS, the Global Fund, International AIDS Society and the WHO. Additionally, we will identify and contact key scholars who examine HIV in sub-Saharan African countries on possible reports and sources of articles that examine stigma and unidentified by the team through other search strategies. Finally, we will supplement our searches of traditional bibliographic databases by searching Google Scholar and screening results until the Google Scholar relevance ranking order shows us 20 results that are not relevant.

\section{Data screening}

Studies will be imported into EndNote and duplicates will be removed. The remaining citations and abstracts, as well as inclusion and exclusion criteria, will be added to Covidence Systematic Review Software. ${ }^{34}$ Using Covidence Systematic Review Software, four reviewers will screen the title and abstract of the first 50 articles in pairs, as recommended in the PRISMA-ScR guideline, to ensure clarity of the inclusion and exclusion criteria. ${ }^{29}$ Inter-rater reliability (IRR) will be estimated using Cohen's kappa. Acceptable agreement will be considered at $\mathrm{k}=0.80 .^{35}$ If we cannot determine study eligibility from the title and abstract alone, the full text will be screened to determine eligibility. After IRR is determined, each of the remaining articles will be reviewed by two independent reviewers. Disagreements will be resolved by GM'RA, DD or AO.

\section{Data extraction \\ Content}

After screening articles for eligibility, we aim to extract the data on publication information, conceptualisation components, methodology and results. On publication information, we will extract author names, year of publication, the journal of publication and funding source, when applicable. On conceptualisation components, we will extract information on the research questions or hypothesis, theoretical or conceptual frameworks used and variables (eg, types of stigma measured, condom use, sex episodes). Concerning the methodology, we will extract information on the study design (eg, qualitative design, mixed-method design, quantitative design), sampling and recruitment techniques (eg, simple random sampling, snowball sampling) and methods of data collection (eg, interviews, surveys, focus group discussion). We will also extract information on the analytical techniques (eg, correlation, qualitative coding). Additionally, we will 
extract data on the setting of the study (eg, country, cardinal point location, rural/urban areas, if identified). For the results, we will first extract the participant information (MSM, WSW, number of participants, socioeconomic characteristics, such as age range, income, education). We will then extract findings of the study on intersectional stigma and its impact on HIV prevention and care in the population. For each finding, we will extract information, such as main thematic areas, quotes, measures of central tendency and statistical findings, when applicable. We will not extract data from single-stigma article beyond identifying the type of stigma examined (eg, gender, HIV).

\section{Process}

To enable a systematic and coordinated approach to data extraction, we will use a data extraction Google Form with questions for each extractor to enter the findings from the study. The tool has specific questions that extractors need to answer. At least two authors will extract data from each article to ensure consistency. The two authors will meet to discuss and evaluate the extracted data for missing information or contradicting reports. Authors GM'RA and LEN will meet with extractors to reach consensus on contradictory information if the primary reviewers could not arrive at a decision.

\section{Analyses and reporting}

We will report the findings from the extracted data based on PRISMA-ScR guidelines. ${ }^{29}$ We will use a table to report the results based on the questions. We will then provide a narrative synthesis of the findings based on the common themes that answer the specific research questions.

\section{Outcome}

The primary outcome of this study is investigating the nature of intersectional stigma associated with HIV risk factors experienced by MSM and WSW living in SubSaharan Africa as well as evaluating the scope of and gaps in existing literature on stigma associated with these populations. The secondary outcome of this study is investigating the impacts of intersectional stigma on access to HIV prevention and care services among our target population.

\section{DISCUSSION}

Sexual minorities in Sub-Saharan African countries, including MSM and WSW, experience various forms of stigma that have effects on their health and well-being. ${ }^{36}$ The intersectional stigma faced by these groups can greatly impact their access to HIV prevention and care services. ${ }^{1437}$ Studying the effect of intersectional stigma in this region is of particular importance because HIV transmission and HIV-related mortality are elevated. ${ }^{38}$ Many interventions have been initiated in this region to expand access to HIV treatment and prevention services, ${ }^{39}$ but a limited number of studies have investigated the role of that intersectional stigma might play in limiting access to these interventions. While there are many studies from a variety of disciplines that have established social determinants of health such as social-economic status, race and sex can privilege or disadvantage people at the individual and societal level, ${ }^{32}$ there has been limited investigation on the manner in which intersectional stigma might privilege or disadvantage people and have effects on the HIV burden in this region. Specifically, more research is needed to understand and to reduce the intersectional stigma creating barriers to accessing HIV services.

This scoping review will contribute important knowledge to HIV prevention, but it is not without limitations. Its specificity to the Sub-Saharan African region may reduce its generalisability to other parts of the world. Nevertheless, given intersectional stigma exists across geopolitical contexts and this scoping review includes an extensive search on current literature, we believe that the knowledge it generates could be applied globally.

\section{Ethical consideration}

Considering that we will not engage patients or the public in the scoping review, we do not need to seek ethical approval from our Institutional Review Board.

\section{Dissemination plan}

We will publish the scoping review findings in a peerreviewed journal to ensure that other scholars, practitioners and the general public can access it for use. We will also present the findings at HIV and stigma conferences and other spaces when the opportunity arises. Additionally, we will use the findings to conceptualise new studies to fill in the scholarly gaps identified.

\section{Author affiliations}

${ }^{1}$ Center for Interdisciplinary Research on AIDS, Yale University School of Public Health, New Haven, Connecticut, USA

${ }^{2}$ Yale University School of Nursing, New Haven, Connecticut, USA

${ }^{3}$ St Michael's Hospital Centre for Urban Health Solutions, Toronto, Ontario, Canada ${ }^{4}$ Center for Interdisciplinary Research on AIDS, New Haven, Connecticut, USA

${ }^{5}$ Yale University School of Medicine, New Haven, Connecticut, USA

${ }^{6} 37$ Military Hospital, Accra, Ghana

${ }^{7}$ Harvey Cushing/John Hay Whitney Medical Library, Yale University, New Haven, Connecticut, USA

${ }^{8}$ Global Health Division, International Development Group RTI, Internation, New York, New York, USA

Acknowledgements This publication was also made possible through core services and support from the Yale University Center for Interdisciplinary Research on AIDS (P30 MH062294) and the Yale AIDS Prevention Training Program (T32MH020031).

Contributors GM'RA is the first and corresponding author; GM'RA, LEN and LN conceived the study; GM'RA, DD, JN, DT, LT, KN and LEN conducted initial review, finalised the focus of the study and designed the study; KN, GM'RA and DD established the search strategy; all authors contributed to drafting the manuscript; GM'RA and DD performed critical revisions of the manuscript. All authors approved the final version of the manuscript.

Funding This study was made possible with funding from the National Institutes of Health, National Institute of Nursing Research, Multi-Level Intervention Addressing Intersectional Stigma to Improve HIV Testing in MSM (R01 NR019009). We thank the Behavioural Insights Team at the Yale Institute of Global Health for their support in the preparation of our data extraction form.

Competing interests None declared. 
Patient consent for publication Not required.

Provenance and peer review Not commissioned; externally peer reviewed.

Supplemental material This content has been supplied by the author(s). It has not been vetted by BMJ Publishing Group Limited (BMJ) and may not have been peer-reviewed. Any opinions or recommendations discussed are solely those of the author(s) and are not endorsed by BMJ. BMJ disclaims all liability and responsibility arising from any reliance placed on the content. Where the content includes any translated material, BMJ does not warrant the accuracy and reliability of the translations (including but not limited to local regulations, clinical guidelines, terminology, drug names and drug dosages), and is not responsible for any error and/or omissions arising from translation and adaptation or otherwise.

Open access This is an open access article distributed in accordance with the Creative Commons Attribution Non Commercial (CC BY-NC 4.0) license, which permits others to distribute, remix, adapt, build upon this work non-commercially, and license their derivative works on different terms, provided the original work is properly cited, appropriate credit is given, any changes made indicated, and the use is non-commercial. See: http://creativecommons.org/licenses/by-nc/4.0/.

\section{ORCID iD}

Gamji M'Rabiu Abubakari http://orcid.org/0000-0002-1718-0381

\section{REFERENCES}

1 James SL, Abate D, Abate KH, et al. Global, regional, and national incidence, prevalence, and years lived with disability for 354 diseases and injuries for 195 countries and territories, 1990-2017: a systematic analysis for the global burden of disease study 2017 . The Lancet 2018;392:1789-858.

2 Roth GA, Abate D, Abate $\mathrm{KH}$, et al. Global, regional, and national age-sex-specific mortality for 282 causes of death in 195 countries and territories, 1980-2017: a systematic analysis for the global burden of disease study 2017. The Lancet 2018;392:1736-88.

3 UN Joint Program on AIDS (UNAIDS). The gap report. Geneva United Nations; 2014.

4 Baral S, Sifakis F, Cleghorn F, et al. Elevated risk for HIV infection among men who have sex with men in low- and middle-income countries 2000-2006: a systematic review. PLoS Med 2007;4:e339.

5 Beyrer C, Baral SD, van Griensven F, et al. Global epidemiology of HIV infection in men who have sex with men. The Lancet 2012;380:367-77.

6 Tat SA, Marrazzo JM, Graham SM. Women who have sex with women living in low- and middle-income countries: a systematic review of sexual health and risk behaviors. LGBT Health 2015;2:91-104.

7 Poteat TC, Logie CH, Adams D, et al. Stigma, sexual health, and human rights among women who have sex with women in Lesotho. Reprod Health Matters 2015;23:107-16.

8 Marrazzo JM. Sexually transmitted infections in women who have sex with women: who cares? Sex Transm Infect 2000;76:330-2.

9 Samuel K. MSM living in African countries that criminalise gay sex are at a much higher risk of getting HIV, 2020. Available: https:// www.aidsmap.com/news/jul-2020/msm-living-african-countriescriminalise-gay-sex-are-much-higher-risk-getting-hiv

10 Croome N, Ahluwalia M, Hughes LD, et al. Patient-reported barriers and facilitators to antiretroviral adherence in Sub-Saharan Africa. AIDS 2017;31:995-1007.

11 National AIDS Trust, FCB Health Network. "Tackling HIV stigma: what works? using the global evidence base to reduce the impact of HIV stigma. ", 2016. https://www.nat.org.uk/publication/tackling-hivstigma-what-works

12 Baral S, Logie CH, Grosso A, et al. Modified social ecological model: a tool to guide the assessment of the risks and risk contexts of HIV epidemics. BMC Public Health 2013;13:482

13 Stangl A, Brady L, Fritz K. Strive technical brief: measuring HIV stigma and discrimination; international center for research on women. Washington DC, USA, 2018.

14 Turan JM, Elafros MA, Logie $\mathrm{CH}$, et al. Challenges and opportunities in examining and addressing intersectional stigma and health. BMC Med 2019;17:7.

15 Bowleg L. The problem with the phrase women and minorities: intersectionality-an important theoretical framework for public health. Am J Public Health 2012;102:1267-73.
16 Sangaramoorthy T, Jamison AM, Dyer TV. HIV stigma, retention in care, and adherence among older black women living with HIV. $J$ Assoc Nurses AIDS Care 2017;28:518-31.

17 Jackson-Best F, Edwards N. Stigma and intersectionality: a systematic review of systematic reviews across HIV/AIDS, mental illness, and physical disability. BMC Public Health 2018;18.

18 Mburu G, Ram M, Siu G, et al. Intersectionality of HIV stigma and masculinity in eastern Uganda: implications for involving men in HIV programmes. BMC Public Health 2014;14.

19 Hargreaves JR, Busza J, Mushati P, et al. Overlapping HIV and sexwork stigma among female sex workers recruited to 14 respondentdriven sampling surveys across Zimbabwe, 2013. AIDS Care 2017;29:675-85.

20 Gu LY, Zhang N, Mayer KH, et al. Autonomy-supportive healthcare climate and HIV-related stigma predict linkage to HIV care in men who have sex with men in Ghana, West Africa. J Int Assoc Provid AIDS Care 2021;20:2325958220978113.

21 Kushwaha S, Lalani Y, Maina G, et al. "But the moment they find out that you are MSM...": a qualitative investigation of HIV prevention experiences among men who have sex with men (MSM) in Ghana's health care system. BMC Public Health 2017;17:770.

22 Kennedy CE, Baral SD, Fielding-Miller R, et al. "They are human beings, they are Swazi": intersecting stigmas and the positive health, dignity and prevention needs of HIV-positive men who have sex with men in Swaziland. J Int AIDS Soc 2013;16:18749.

23 Cloete A, Simbayi LC, Kalichman SC, et al. Stigma and discrimination experiences of HIV-positive men who have sex with men in Cape town, South Africa. AIDS Care 2008;20:1105-10.

24 Anderson AM, Ross MW, Nyoni JE, et al. High prevalence of stigma-related abuse among a sample of men who have sex with men in Tanzania: implications for HIV prevention. AIDS Care 2015;27:63-70.

$25 \mathrm{Kim} \mathrm{H}-\mathrm{Y}$, Grosso A, Ky-Zerbo O, et al. Stigma as a barrier to health care utilization among female sex workers and men who have sex with men in Burkina Faso. Ann Epidemiol 2018;28:13-19.

26 Bwambale FM, Ssali SN, Byaruhanga S, et al. Voluntary HIV counselling and testing among men in rural Western Uganda: implications for HIV prevention. BMC Public Health 2008;8:263-76.

27 Nelson LE, McMahon J, Zhang N. Exploring HIV, same-sex and gender non-conformity stigmas and delays in HIV diagnosis, linkage and retention for MSM in Ghana. National Institute of Mental Health, 2016.

28 Holtzman S, Landis L, Walsh Z, et al. Predictors of HIV testing among men who have sex with men: a focus on men living outside major urban centres in Canada. AIDS Care 2016;28:705-11.

29 Tricco AC, Lillie E, Zarin W, et al. PRISMA extension for scoping reviews (PRISMA-ScR): checklist and explanation. Ann Intern Med 2018;169:467-73.

30 Yale University Institutional Review Boards. IRB policy 100 institutional review board (IRB) review of human subject research protocols or FDA- regulated activities involving human participants. New Haven: USA, 2018.

31 Mburu G, Igbinedion E, Lim SH, et al. Outcomes of HIV treatment from the private sector in low-income and middle-income countries: a systematic review protocol. BMJ Open 2020;10:e031844.

32 UNICEF. The state of the world's ch ildren 1996 report. New York, USA; 1996. https://www.unicef.org/sowc96/groups.htm

33 Grossman AH. Gay men and HIV/AIDS: understanding the double stigma. J Assoc Nurses AIDS Care 1991;2:28-32.

34 . Covidence systematic review software. Melbourne, Australia Veritas Health Innovation; 2019. www.covidence.org

35 Belur J, Tompson L, Thornton A, et al. Interrater reliability in systematic review methodology: exploring variation in coder decision-making. Sociol Methods Res 2021;50:837-65.

36 Risher K, Adams D, Sithole B, et al. Sexual stigma and discrimination as barriers to seeking appropriate healthcare among men who have sex with men in Swaziland. J Int AIDS Soc 2013;16:18715.

37 Eichenberger A, Weisser M, Battegay M. [HIV in Sub-Saharan Africa: where are we today?]. Praxis 2019;108:971-6.

38 Kharsany ABM, Karim QA. HIV infection and AIDS in sub-Saharan Africa: current status, challenges and opportunities. Open AIDS J 2016;10:34-48.

39 Ortblad KF, Baeten JM, Cherutich P, et al. The Arc of HIV epidemics in sub-Saharan Africa: new challenges with concentrating epidemics in the era of 90-90-90. Curr Opin HIV AIDS 2019;14:354-65. 